\title{
Obesity and risk of subsequent hospitalisation with pneumonia
}

\author{
J.B. Kornum*, M. Nørgaard*, C. Dethlefsen\#, K.M. Due ${ }^{\#}$, R.W. Thomsen*, \\ A. Tjønneland ${ }^{\oplus}$, H.T. Sørensen* and K. Overvad ${ }^{*}, \#,+$
}

ABSTRACT: Obesity may be associated with increased risk of pneumonia, but available data on this relationship are sparse and inconsistent.

We followed a prospective cohort of 22,578 males and 25,973 females from the Danish Diet, Cancer and Health Study, aged 50-64 yrs and free from major chronic diseases at baseline (19931997), for first-time hospitalisation with pneumonia (median follow-up 12 yrs).

Compared with males of normal weight, adjusted hazard ratios (HRs) for pneumonia were 1.4 (95\% Cl 1.2-1.7) for males with moderate obesity (body mass index (BMI) $30.0-34.9 \mathrm{~kg} \cdot \mathrm{m}^{-2}$ ), and $2.0(95 \% \mathrm{Cl} 1.4-2.8)$ for males with severe obesity $\left(B M I \geqslant 35.0 \mathrm{~kg} \cdot \mathrm{m}^{-2}\right.$ ), controlling for lifestyle and educational variables. Among females the associations were weaker, with adjusted HRs of 0.8 (95\% Cl 0.6-1.0) for moderate obesity, and 1.2 (95\% Cl 0.8-1.6) for severe obesity. Adjustment for major chronic diseases diagnosed during follow-up eliminated the associations between obesity and pneumonia risk.

Obesity is associated with higher risk of hospitalisation with pneumonia among males but not among females, which is apparently explained by occurrence of other chronic diseases.

KEYWORDS: Body mass index, chronic diseases, epidemiological study, obesity, pneumonia, risk

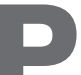

neumonia is an important cause of morbidity, mortality and cost of care worldwide. Combined with influenza, pneumonia is the eighth leading cause of death in the USA and the most frequent cause of death due to infectious disease [1]. During the past decade, hospitalisations with pneumonia have increased by $20-50 \%$ in Western populations [2-4]. Ageing populations and an increase in chronic conditions such as heart disease, diabetes, chronic obstructive pulmonary disease and cancer have been suggested as important factors underlying this increasing trend [2-4]. At the same time, the prevalence of obesity has increased worldwide [5].

Obese individuals may have an increased risk of respiratory tract infections, including pneumonia, but the few recent studies on this topic gave conflicting results [6-8]. Recently, obesity has also been related to severe episodes of influenza A (H1N1) [9] and to presence of bacterial pneumonia in fatal influenza A (H1N1) cases [10]. Obese individuals may have increased susceptibility to lower respiratory tract infection due to impaired T- and B-cell-mediated immune responses, a higher risk of aspiration, reduced lung volume, and an altered ventilation pattern [11-13]. Obesity is also related to other major chronic diseases that may increase the risk of pneumonia, including diabetes, heart failure, stroke, asthma, gastro-oesophageal reflux, certain forms of cancer, and liver disease $[5,13]$. It is unclear whether any relationship between obesity and pneumonia risk may be due to obesity per se or explained by comorbidities induced by obesity. Furthermore, the risk from obesity may differ according to sex [7].

Additional studies are needed to identify risk groups for severe pneumonia and to qualify the current debate on the appropriateness of influenza and pneumococcal immunisations in individuals with obesity [9]. In this prospective cohort study we examined whether obesity (measured by body mass index (BMI)) is associated with the risk of pneumonia-related hospitalisation among males and females and to examine whether such association is explained by the occurrence of other chronic diseases in obese individuals.

\section{MATERIALS AND METHODS}

\section{Study population}

We conducted this cohort study using data from the Danish Diet, Cancer and Health Study [14]. From December 1993 to May 1997, a total of 160,725 individuals (80,996 males and 79,729 females) were invited to participate in the study.
AFFILIATIONS

*Dept of Clinical Epidemiology,

Aarhus University Hospital,

\#Dept of Clinical Epidemiology,

Center for Cardiovascular Research,

Aalborg Hospital, Aarhus University Hospital,

${ }^{+}$Dept of Cardiology, Aalborg

Hospital, Aarhus University Hospital,

Aarhus, and

-Danish Cancer Society,

Copenhagen, Denmark.

CORRESPONDENCE

J.B. Kornum

Dept of Clinical Epidemiology

Sdr. Skovvej 15

DK-9100 Aalborg

Denmark

E-mail: j.kornum@rn.dk

Received:

Nov 192009

Accepted after revision:

March 202010

First published online:

March 292010 
Its overall aim was to examine the association between specific dietary components, foods and nutrients, and risk of cancer, obesity and chronic diseases. Eligible participants were 5064 yrs of age, born in Denmark, inhabitants of Copenhagen or Aarhus, and had no record of cancer in the Danish Cancer Registry. A total of 57,053 individuals (27,178 males and 29,875 females) were enrolled in the study. All participants completed a detailed food frequency questionnaire and a second baseline questionnaire on lifestyle and background. Anthropometric measurements were obtained at baseline.

For the purposes of our analysis, we excluded 826 individuals who had experienced hospitalisation for pneumonia before enrolment, 337 individuals because of a missing baseline questionnaire or missing variables, and an additional 7,339 individuals who had other major chronic diseases diagnosed before enrolment (see below for definition of chronic diseases). Thus, data for a total of 48,551 individuals (22,578 males and 25,973 females) were included in our analysis.

The Danish National Health Service provides universal taxsupported healthcare, including free access to primary and hospital care. Since 1968, all Danish residents have received a unique civil registration number, encoding sex and date of birth, which is used in all health registries and permits unambiguous registry record linkage.

\section{Anthropometry}

Anthropometric measurements were collected at two study clinics in Aarhus and Copenhagen, Denmark. Height was measured with participants standing without shoes and recorded to the nearest $0.5 \mathrm{~cm}$. Weight was measured using a digital scale with participants wearing light clothing or underwear, and recorded to the nearest $100 \mathrm{~g}$. BMI was calculated as weight in kilograms divided by the square of the height in metres.

\section{Identification of first-time hospitalisation with pneumonia}

We identified study participants with a first-time pneumoniarelated hospitalisation using the discharge diagnosis codes in the Danish National Registry of Patients. This registry has collected data on all hospitalisations in Denmark since 1977 and on all outpatient visits since 1995, and includes civil registration number, surgical procedures, and up to 20 discharge diagnoses. Diagnoses are coded by physicians according to the International Classification of Diseases, 8th revision (ICD-8), until the end of 1993, and 10th revision (ICD-10) thereafter. We identified all hospital discharges with a diagnosis of pneumonia (ICD-10 codes J12.x-J18.x), ornithosis (ICD-10 code A709.x), or legionellosis (ICD-10 code A481.x) occurring between the date of enrolment into the study cohort and April 10, 2008. Patients who had been hospitalised for pneumonia before study enrolment (with ICD-10 codes above or ICD-8 codes 480.XX-486.XX, 0.73.XX, and 471.XX) were excluded.

\section{Information on death}

We ascertained information regarding death from the Danish Civil Registration System.

\section{Potential confounders}

Lifestyle and educational factors

In the food frequency questionnaire, alcohol intake (beer, wine, fortified wine and spirits) was reported as the average amount consumed over the preceding year. Total alcohol intake was converted into $\mathrm{g} \cdot \mathrm{day}^{-1}$. From the lifestyle questionnaire, we obtained information on smoking status, schooling and educational level.

\section{Other major chronic diseases}

Data on other major chronic diseases diagnosed during followup were collected from the Danish National Registry of Patients using hospital discharge or hospital outpatient visit diagnoses. For diabetes, we additionally obtained data from self-reports and from the Danish National Diabetes Register, which contains information on all individuals with recorded diabetes in Denmark between 1996 and 2006 [15]. To control for a variety of chronic diseases diagnosed during follow-up, we computed an extended version of the summary measure of comorbidity developed by CHARLSON et al. [16]. The original Charlson Index includes 19 major disease categories, including congestive heart failure, chronic pulmonary disease, cerebrovascular disease, dementia, diabetes, renal disease, cancer, liver disease and AIDS [16], all of which are known to increase the risk of pneumonia. We added diagnoses of HIV without AIDS to the index, and also added diagnoses of gastrooesophageal reflux and hypertension with 1 point each. We then computed the cumulative comorbidity index score achieved during follow-up and up to the first pneumonia-related hospitalisation for each participant. Three comorbidity levels were defined: low (score of 0$)$, medium $(1-2)$, and high $(\geqslant 3)$.

\section{Statistical analysis}

We divided the participants according to BMI $(<22.5,22.5$ $24.9,25.0-29.9,30.0-34.9$ and $\geqslant 35 \mathrm{~kg} \cdot \mathrm{m}^{-2}$ ) similar to groupings used by the World Health Organization. Because of the few participants with $\mathrm{BMI}<18.5 \mathrm{~kg} \cdot \mathrm{m}^{-2}$, we used a different cutpoint for the lowest BMI category. We defined normal weight as BMI $22.5-24.9 \mathrm{~kg} \cdot \mathrm{m}^{-2}$, overweight as BMI $25.0-29.9 \mathrm{~kg} \cdot \mathrm{m}^{-2}$, moderate obesity as BMI $30.0-34.9 \mathrm{~kg} \cdot \mathrm{m}^{-2}$ and severe obesity as $\mathrm{BMI} \geqslant 35.0 \mathrm{~kg} \cdot \mathrm{m}^{-2}$. We used age as the underlying time variable, with follow-up starting at age at study enrolment. Follow-up extended until age on April 10, 2008, or until age at pneumonia diagnosis, death or migration, whichever occurred first. We computed pneumonia incidence rates by dividing the number of events by the accumulated person-time of follow-up within the groups of BMI, separately for males and females. Stratified analyses were performed by age (50-54, 55-59 and 60 yrs) and current smoking status (current smokers and nonsmokers). Cox's regression was used to compute hazard ratios (HRs) as a measure of relative risk for hospitalisation with pneumonia, according to BMI (reference BMI $22.5-24.9 \mathrm{~kg} \cdot \mathrm{m}^{-2}$ ). We controlled for baseline smoking status (never, former and current $<15,15-25$ or $>25 \mathrm{~g}$ of tobacco per day), alcohol intake (modelled as a restricted cubic spline and an indicator variable for drinking versus non-drinking), schooling (7, 8-10 and $>10 \mathrm{yrs}$ ) and educational level (no education, short, middle and long). The relationships between BMI and pneumonia risk were also illustrated with the use of restricted cubic splines (reference BMI $22.5 \mathrm{~kg} \cdot \mathrm{m}^{-2}$ ). Next, we examined whether any association between obesity and pneumonia was explained by occurrence of comorbidities, by adding the level of the extended Charlson index score achieved during follow-up and before a first episode of pneumonia-related hospitalisation as a time-dependent variable (score level low, medium and high) in 
the regression model. Finally, we examined the association between obesity and risk of hospitalisation with nonfatal and fatal pneumonia; i.e. hospitalisation ending with death $\leqslant 30$ days after admission. Data were analysed with Stata Statistical Software (Release 10.1 SE; State Corporation, College Station, TX, USA). The ethical committee and the Danish Data Protection Agency approved the study.

\section{RESULTS}

\section{Descriptive data}

Among the 48,551 individuals included in our cohort, 1,087 males and 1,025 females had a first episode of pneumoniarelated hospitalisation during a median follow-up period of 11.8 and 11.9 yrs, respectively. The corresponding incidence rates of hospitalisations with pneumonia were 4.2 per 1000 person-years for males and 3.4 per 1,000 person-yrs for females. Table 1 presents characteristics for the participants in the cohort overall and according to BMI. At enrolment, 50\% of the participating males were overweight, $12 \%$ were moderately obese and $2 \%$ were severely obese. Among females, 34\% were overweight, $10 \%$ were moderately obese and $3 \%$ were severely obese. Compared with participants of normal weight, obese participants were less likely to be smokers but more likely to be former smokers, and were on average less educated. Furthermore, slightly more obese males reported high alcohol intake compared with males of normal weight, while obese females reported lower alcohol intake than normal weight females.

\section{Obesity and risk of hospitalisation with pneumonia}

The baseline adjusted HR for hospitalisation with pneumonia (i.e., adjusted for baseline smoking status, alcohol intake, schooling and education) according to BMI is shown as a smoothed curve in figure 1 , and indicates a higher risk of pneumonia among participants with low normal BMI and a higher risk of pneumonia among male overweight and obese participants. The association between BMI and pneumonia risk was only modest among overweight and obese females.

Compared with males of normal weight, baseline adjusted HRs were 1.4 (95\% CI 1.2-1.7) for males with moderate obesity, and 2.0 (95\% CI 1.4-2.8) for males with severe obesity (table 2). Associations between obesity and increased pneumonia risk tended to be strongest in nonsmoking males and in those aged $\geqslant 60$ yrs. Among females the associations were much weaker, with adjusted HRs of 0.8 (95\% CI 0.6-1.0) for females with moderate obesity, and 1.2 (95\% CI 0.8-1.6) for females with severe obesity. The associations were much the same in different age groups, and were strongest among nonsmoking females (table 2). All baseline adjusted HRs were close to the crude estimates, suggesting little confounding by smoking status, alcohol intake, schooling and educational level (table 2).

Among participants with moderate to severe obesity, 48-58\% of males and $41-50 \%$ of females were diagnosed with other major chronic diseases during followup and up to the first pneumonia-related hospitalisation or censoring (table 1). The corresponding proportions were $34 \%$ among normal weight males and $32 \%$ among normal weight females. After adjustment for chronic diseases diagnosed during follow-up, the baseline adjusted HRs were 1.0 (95\% CI 0.8-1.3) for males with moderate obesity, and 1.2 (95\% CI $0.8-1.7)$ for males with severe obesity. When chronic diseases diagnosed during the pneumonia-related hospitalisation were also added to the model, there was no longer any association between pneumonia and severe obesity (adjusted HR 1.0, 95\% CI 0.7-1.5). Among females, after adjustment for chronic diseases diagnosed during follow-up, the HRs were 0.7 (95\% CI 0.6-0.9) for females with moderate obesity, and 0.8 (95\% CI 0.6-1.1) for females with severe obesity.

In total, 108 males and 65 females had a pneumonia-related hospitalisation which led to death within 30 days. When we assessed associations between hospitalisation with fatal pneumonia and obesity among males, the baseline adjusted HRs were imprecise, yet slightly higher than those for non-fatal pneumonia, i.e., 1.7 (95\% CI 0.9-3.3) for fatal versus 1.4 (95\% CI 1.1-1.7) for nonfatal pneumonia in the moderate obesity group and 2.1 (95\% CI 0.6-7.2) for fatal versus. 2.0 (95\% CI 1.4-2.9) for non-fatal pneumonia in the severe obesity group. Among females, the corresponding HRs were 0.8 (95\% CI 0.3-2.1) versus 0.8 (95\% CI $0.6-1.1)$ in the moderate obesity group and 1.8 (95\% CI 0.6-5.5) versus 1.1 (95\% CI 0.8-1.6) in the severe obesity group. All risk increases vanished when adding other chronic diseases diagnosed during follow-up to the adjusted model: fatal pneumonia HRs with moderate and severe obesity were 0.9 (95\% CI 0.5-1.7) and 0.8 (95\% CI 0.2-2.7) for males, and 0.6 (95\% CI 0.2-1.6) and 0.9 (95\% CI 0.3-2.8) for females.

\section{DISCUSSION}

In this large prospective cohort study, conducted among Danes aged 50-64 yrs with no hospital-diagnosed comorbidity at enrolment, we found that moderate obesity was associated with a 1.4-fold higher risk of a pneumonia-related hospitalisation among males, while severe obesity was associated with a 2.0-fold higher risk. Only weak associations were observed among females. Similar associations with obesity were seen with pneumonias that ended in fatality. The higher risk conferred by obesity was apparently explained by occurrence of other major chronic diseases during follow-up.

The results were obtained from the Danish Diet, Cancer and Health cohort study, in which follow-up of the participants was virtually complete. All height and weight measurements were performed by a laboratory technician at enrolment, omitting potential bias caused, for example, by understated weight in obese individuals. If obese participants gained further weight during follow-up, the association between baseline obesity and risk of pneumonia would be overestimated. Physicians may be more likely to hospitalise obese than lean patients with infection. Such bias would also cause overestimation of the relative risk associated with obesity. Still, potentially increased surveillance could not explain the observed differences in risk estimates between males and females. Our study relies on discharge diagnoses, which were not independently confirmed. However, while some errors in coding pneumonia diagnoses may have occurred, the positive predictive value of a pneumonia diagnosis in the National Patient Registry has previously been estimated to be $90 \%$ (95\% CI 82-95\%) [3].

Besides increasing age and presence of comorbidities, smoking and use of alcohol may increase risk of pneumonia [7]. Smoking is associated with low BMI [17], whereas results 
TABLE 1 Characteristics of persons participating in the Danish Diet, Cancer and Health study according to body mass index

Body mass index $\mathrm{kg} \cdot \mathrm{m}^{-2}$

$\begin{array}{llll}<22.5 & 22.5-24.9 & 25.0-29.9 & 30.0-34.9\end{array}$

\section{Males}

Participants $\mathrm{n}$

Pneumonia

Age yrs

$50-54$

55-59

60-64

$\geqslant 65$

Smoking

Never

Former

Current

Alcohol standard drinks per week

$>0-6$

$>6-13$

$>13-21$

$>21$

Schooling yrs

7

$8-10$

$>10$

Education

No

Short

Middle

Long

Comorbidity index ${ }^{\#}$

Low

Medium

High

\section{Females}

Participants $n$

Pneumonia

Age yrs

50-54

55-59

60-64

$\geqslant 65$

Smoking

Never

Former

Current

Alcohol standard drinks per week

$>0-6$

$>6-13$

$>13-21$

$>21$

Schooling yrs

7

$8-10$

$>10$

Education

No

Short

Middle

Long

Comorbidity index ${ }^{\#}$

Low

Medium

High

22578

1087 (5)

8577 (38)

7306 (32)

5469 (24)

$1226(5)$

$6124(27)$

7718 (34)

8736 (39)

334 (1)

5007 (22)

6977 (31)

3571 (16)

$6689(30)$

7483 (33)

$9499(42)$

5596 (25)

2082 (9)

3081 (14)

9497 (42)

7918 (35)

$13940(62)$

6269 (28)

$2369(10)$

25973

1025 (4)

9320 (36)

8337 (32)

6788 (26)

1528 (6)

$11698(45)$

6000 (23)

8275 (32)

599 (2)

$13016(50)$

7358 (28)

2882 (11)

2118 (8)

7816 (30)

13193 (51)

4964 (19)

4797 (18)

8213 (32)

9960 (38)

3003 (12)

17056 (66)

6798 (26)

2119 (8)
2260

$134(6)$

907 (40)

720 (32)

527 (23)

106 (5)

\section{5 (25)}

505 (22)

1180 (52)

44 (2)

540 (24)

668 (30)

$364(16)$

644 (29)

583 (26)

956 (42)

721 (32)

154 (7)

309 (14)

935 (41)

862 (38)

1495 (66)

577 (26)

188 (8)

6472

304 (5)

2675 (41)

2000 (31)

1472 (23)

325 (5)

2643 (41)

1299 (20)

2530 (39)

147 (2)

2887 (45)

1942 (30)

932 (14)

564 (9)

1440 (22)

3354 (52)

1678 (26)

871 (13)

2011 (31)

2627 (41)

963 (15)

4454 (69)

1565 (24)

453 (7)
5879

245 (4)

2257 (38)

1892 (32)

1427 (24)

303 (5)

1643 (28)

1732 (29)

2504 (43)

88 (2)

1279 (22)

1897 (32)

972 (17)

1643 (28)

1591 (27)

2514 (43)

1774 (30)

428 (7)

735 (13)

2448 (42)

2268 (39)

3877 (66)

1519 (26)

483 (8)

7204

283 (4)

2658 (37)

2382 (33)

1773 (25)

391 (5)

3191 (44)

1659 (23)

2354 (33)

123 (2)

3342 (46)

2236 (31)

843 (12)

660 (9)

1930 (27)

3803 (53)

1471 (20)

1127 (16)

2329 (32)

2890 (40)

858 (12)

4913 (68)

1800 (25)

491 (7)
11317

510 (5)

4245 (38)

3666 (32)

2735 (24)

671 (6)

3048 (27)

4250 (38)

4019 (36)

158 (1)

2460 (22)

3524 (31)

1797 (16)

3378 (30)

3887 (34)

4808 (42)

2622 (23)

1057 (9)

1610 (14)

4764 (42)

3886 (34)

7002 (62)

3140 (28)

1175 (10)

8867

309 (3)

2879 (32)

2877 (32)

2560 (29)

551 (6)

4113 (46)

2194 (25)

2560 (29)

197 (2)

4667 (53)

2453 (28)

854 (10)

696 (8)

3011 (34)

4449 (50)

1407 (16)

1833 (21)

2799 (32)

3333 (38)

902 (10)

5754 (65)

2360 (27)

753 (8)

$\begin{array}{cc}2671 & 451 \\ 163(6) & 35(8) \\ 983(37) & 185(41) \\ 871(33) & 157(35) \\ 678(25) & 102(23) \\ 139(5) & 7(2)\end{array}$

726 (27)

1057 (40)

132 (29)

888 (33)

145 (32)

$36(1)$

607 (23)

767 (29)

388 (15)

873 (33)

8 (2)

121 (27)

121 (27)

50 (11)

151 (33)

1181 (44) $241(53)$

$1061(40) \quad 160(35)$

$429(16) \quad 50(11)$

359 (13) $\quad 84(19)$

$357(13) \quad 70(16)$

$1175(44) \quad 175(39)$

$780(29) \quad 122(27)$

$1378(52) \quad 188(42)$

861 (32) $\quad 172$ (38)

432 (16) $\quad 91(20)$ 

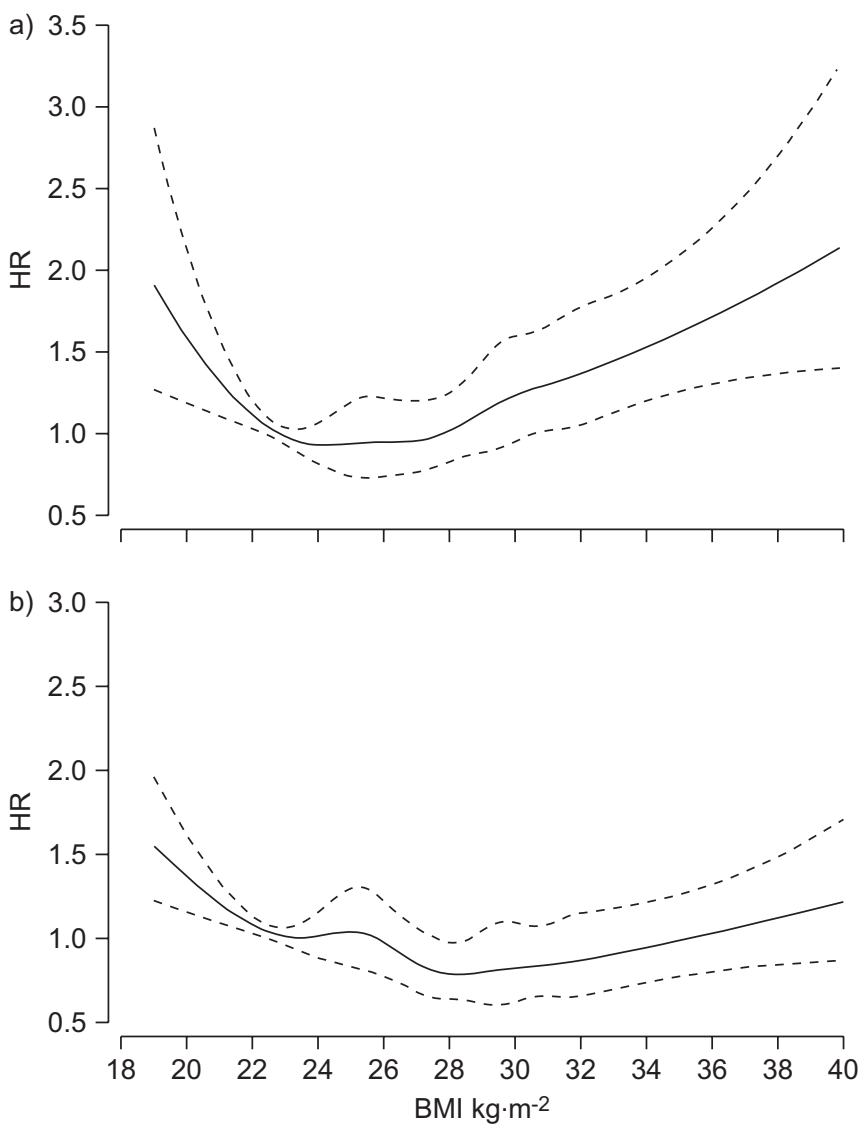

FIGURE 1. Adjusted hazard ratio (HR) for hospitalisation with pneumonia among males and females in the Danish Diet, Cancer and Health Study according to body mass index (BMI). The HR for hospitalisation with pneumonia in association with $\mathrm{BMI}$ is shown for a) males and b) females. -: HR; ---: 95\% Cls derived from restricted cubic spline regression with seven knots. The reference point for BMl is $22.5 \mathrm{~kg} \cdot \mathrm{m}^{-2}$. Age was used as the underlying time variable in the regression model with additional adjustment for smoking status, alcohol intake, schooling and educational level.

from studies on alcohol intake and body weight are inconsistent [18]. As we based our information on self-reported smoking and alcohol use at the time of enrolment, we cannot exclude residual confounding if this information was wrongly reported, or if smoking and drinking habits changed over time. Still, alcohol use and smoking did not act as strong confounders in our study. Although the Charlson index has been validated for prediction of mortality rather than for prediction of subsequent diseases [16], our extended comorbidity index enabled us to adjust simultaneously for a wide range of important diseases acting as risk factors for pneumonia. Still, unknown or unmeasured confounders including use of medications may have affected our risk estimates.

It has been debated as to which obesity markers are most appropriate for assessing the risk of subsequent disease [19]. We used BMI as an indirect measure of weight and obesity. The BMI does not, however, take age, sex, bone structure, fat distribution or muscle mass into consideration [20]. Nevertheless, BMI is easily obtainable and allowed us to compare our findings with the results of previous studies on this topic.
Earlier studies on pneumonia risk in obese individuals yielded conflicting findings [6-8]. Among US males from the Health Professionals' Follow-up Study and US females from the Nurses' Health Study II, who were free of asthma, cardiovascular disease, cancer, and diabetes at baseline, BAIK et al. [7] found that a BMI $\geqslant 30 \mathrm{~kg} \cdot \mathrm{m}^{-2}$ was associated with a higher risk of pneumonia among females (adjusted relative risk 2.2, 95\% CI 1.6-3.2) but not among males (adjusted relative risk 1.0, 95\% CI 0.6-1.7). Compared with our study, the US cohorts included younger females (aged 27-44 yrs) and older males (aged 44$79 \mathrm{yrs}$ ). If the relative impact of obesity is greater in younger than older adults, age differences might explain part of the diverging results. Furthermore, the Nurses' Health Study II was based on self-reported episodes of physician-diagnosed pneumonia with the risk of some over- or under-reporting, while we used independent population-based medical databases to obtain information on pneumonia hospitalisations. Finally, chronic diseases occurring during follow-up were not taken into account in the US study. In a Spanish case-control study based on pneumonia cases and age-, sex- and primary centre-matched controls, Almirall et al. [6] reported a slightly lower risk of pneumonia among obese individuals in univariate analysis (OR 0.8, 95\% CI 0.6-1.0). A similar lower risk was also found in multivariate analysis among obese individuals in a smaller German case-control study (OR 0.7, 95\% 0.5-0.9) [8]. Insufficient adjustment for smoking among lean individuals may have contributed to these findings. Conversely, compared with the cohort studies, case-control studies may have the advantage of measuring BMI close to the index pneumonia episode, thus avoiding overestimation of any association through bias introduced by additional weight gain over time.

After we added other major chronic diseases to the regression model, obesity no longer predicted risk of pneumonia, and the risk even tended to be lower among females. Thus, our analysis suggests that most of the higher pneumonia risk associated with obesity is explained by the occurrence of other chronic diseases. Obesity is strongly associated with type 2 diabetes, and diabetes has been associated with a $25-75 \%$ higher risk of hospitalized pneumonia episodes [21], possibly because of the harmful effects of hyperglycaemia on the immune system, increased risk of aspiration due to diabetic gastroparesis, and impaired lung function [22]. A metaanalysis of seven prospective cohort studies showed that obesity and being overweight are associated with a 1.5-fold greater risk of asthma [23]. Asthma is a risk factor for pneumonia [24]. Obesity also increases the risk of both ischaemic heart disease and chronic heart failure [5], and in particular, the latter has been associated with a doubled risk of pneumonia-related hospitalisations [25]. Other diseases, such as gastro-oesophageal reflux, stroke and dementia, increase the likelihood of pneumonia through aspiration. Finally, obesity is associated with chronic renal disease through associated hypertension and diabetes, and immune cell dysfunction is known to occur among patients with chronic renal failure and haemodialysis [22].

We found that obesity was much more strongly related to the risk of pneumonia among males than among females. The sex differences weakened, but remained present, after adjustment for other major chronic diseases. The reasons for this striking 
TABLE 2 Incidence rates and hazard ratios (HRs) for hospitalisation with pneumonia among males and females according to body mass index

Body mass index $\mathbf{k g} \cdot \mathrm{m}^{-2}$

\begin{tabular}{|c|c|c|c|c|c|}
\hline & \multicolumn{5}{|c|}{ Body mass index $\mathrm{kg} \cdot \mathrm{m}^{-2}$} \\
\hline & $<22.5$ & $22.5-24.9$ & $25.0-29.9$ & $30.0-34.9$ & $\geqslant 35$ \\
\hline \multicolumn{6}{|l|}{ Males } \\
\hline Incidence rate per 1,000 person-yrs & 5.2 & 3.6 & 3.9 & 5.4 & 7.0 \\
\hline \multicolumn{6}{|l|}{ Overall HR $(95 \% \mathrm{Cl})$} \\
\hline Adjusted $^{+}$ & $1.4(1.1-1.7)$ & 1.0 (ref.) & $1.0(0.8-1.1)$ & $1.0(0.8-1.3)$ & $1.2(0.8-1.7)$ \\
\hline \multicolumn{6}{|l|}{ HR (95\% Cl) by subgroup } \\
\hline \multicolumn{6}{|l|}{ Age yrs } \\
\hline $50-54$ & $1.3(0.8-1.9)$ & 1.0 (ref.) & $1.0(0.8-1.4)$ & $1.6(1.1-2.3)$ & $1.4(0.7-2.9)$ \\
\hline $55-59$ & $1.2(0.8-1.7)$ & 1.0 (ref.) & $0.8(0.6-1.1)$ & $1.2(0.9-1.7)$ & $2.0(1.2-3.5)$ \\
\hline$\geqslant 60$ & $1.7(1.2-2.4)$ & 1.0 (ref.) & $1.4(1.1-1.7)$ & $1.5(1.1-2.1)$ & $2.3(1.3-4.3)$ \\
\hline \multicolumn{6}{|l|}{ Smoking status ${ }^{\S}$} \\
\hline \multicolumn{6}{|l|}{ Females } \\
\hline Incidence rate per 1,000 person-yrs & 4.0 & 3.4 & 3.0 & 2.9 & 4.3 \\
\hline \multicolumn{6}{|l|}{ Overall HR $(95 \% \mathrm{Cl})$} \\
\hline Crude $^{\#}$ & $1.3(1.1-1.5)$ & 1.0 (ref.) & $0.8(0.7-1.0)$ & $0.8(0.6-1.0)$ & $1.2(0.8-1.6)$ \\
\hline Adjusted" & $1.2(1.0-1.4)$ & 1.0 (ref.) & $0.9(0.7-1.0)$ & $0.8(0.6-1.0)$ & $1.2(0.8-1.6)$ \\
\hline Adjusted $^{+}$ & $1.2(1.0-1.4)$ & 1.0 (ref.) & $0.8(0.7-1.0)$ & $0.7(0.6-0.9)$ & $0.8(0.6-1.1)$ \\
\hline \multicolumn{6}{|l|}{ HR $(95 \% \mathrm{Cl})$ by subgroup } \\
\hline \multicolumn{6}{|l|}{ Age yrs } \\
\hline 50-54 & $1.3(0.9-1.8)$ & 1.0 (ref.) & $0.8(0.6-1.2)$ & $0.9(0.6-1.5)$ & $1.1(0.5-2.4)$ \\
\hline $55-59$ & $1.2(0.9-1.6)$ & 1.0 (ref.) & $0.9(0.7-1.2)$ & $0.8(0.5-1.2)$ & $1.3(0.8-2.4)$ \\
\hline$\geqslant 60$ & $1.2(0.9-1.5)$ & 1.0 (ref.) & $0.8(0.7-1.1)$ & $0.8(0.6-1.2)$ & $1.1(0.7-1.8)$ \\
\hline \multicolumn{6}{|l|}{ Smoking status ${ }^{\S}$} \\
\hline
\end{tabular}

\#: the crude HR was calculated with the use of Cox's regression, with age as the underlying time variable; ": multivariable-adjusted model was based on the crude model with additional adjustment for smoking status, alcohol intake, schooling and educational level; ${ }^{+}$: multivariable-adjusted model as specified above and with additional

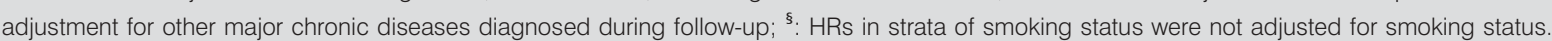

difference are unclear. Differences in fat distribution may play an important role, as abdominal obesity, being more prevalent in males than in females, may restrict the descent of the diaphragm and cause reduced ventilation at the lung bases.

The focus of our study was obesity, yet, as an additional finding, we observed that low BMI was associated with a higher risk of pneumonia in both males and females, which accords with previous studies $[6,8]$. The association with low BMI was absent among nonsmokers in our study, which suggests that the increased pneumonia risk among individuals with low BMI may be largely explained by residual confounding by smoking. Also, a low BMI could be a marker for malnutrition, undiagnosed subclinical conditions, or both.

In conclusion, our data showed that obesity is associated with a markedly higher risk of subsequent hospitalisation with pneumonia among males but not among females. This higher risk is apparently explained by presence of other chronic diseases in obese individuals.

\section{SUPPORT STATEMENT}

This work was supported by Klinisk Epidemiologisk Forskningsfond at Aarhus University and by the Danish Obesity Research Center.

\section{STATEMENT OF INTEREST}

None declared.

\section{REFERENCES}

1 Kung HC, Hoyert DL, Xu J, et al. Deaths: final data for 2005. Natl Vital Stat Rep 2008; 56: 1-120.

2 Fry AM, Shay DK, Holman RC, et al. Trends in hospitalizations for pneumonia among persons aged 65 years or older in the United States, 1988-2002. JAMA 2005; 294: 2712-2719.

3 Thomsen RW, Riis A, Nørgaard M, et al. Rising incidence and persistently high mortality of hospitalized pneumonia: a 10-year population-based study in Denmark. J Intern Med 2006; 259: 410-417.

4 Trotter CL, Stuart JM, George R, et al. Increasing hospital admissions for pneumonia, England. Emerg Infect Dis 2008; 14: 727-733. 
5 Formiguera X, Canton A. Obesity: epidemiology and clinical aspects. Best Pract Res Clin Gastroenterol 2004; 18: 1125-1146.

6 Almirall J, Bolibar I, Serra-Prat M, et al. New evidence of risk factors for community-acquired pneumonia: a population-based study. Eur Respir J 2008; 31: 1274-1284.

7 Baik I, Curhan GC, Rimm EB, et al. A prospective study of age and lifestyle factors in relation to community-acquired pneumonia in US men and women. Arch Intern Med 2000; 160: 3082-3088.

8 Schnoor M, Klante $\mathrm{T}$, Beckmann M, et al. Risk factors for community-acquired pneumonia in German adults: the impact of children in the household. Epidemiol Infect 2007; 135: 1389-1397.

9 Vaillant L, La RG, Tarantola A, et al. Epidemiology of fatal cases associated with pandemic H1N1 influenza 2009. Euro Surveill 2009; 14: 19309.

10 Centers for Disease Control and Prevention, Bacterial coinfections in lung tissue specimens from fatal cases of 2009 pandemic influenza A (H1N1) - United States, May-August 2009. MMWR Morb Mortal Wkly Rep 2009; 58: 1071-1074.

11 Chandra RK. Immune response in overnutrition. Cancer Res 1981; 41: 3795-3796.

12 Falagas ME, Kompoti M. Obesity and infection. Lancet Infect Dis 2006; 6: 438-446.

13 Kopelman PG. Obesity as a medical problem. Nature 2000; 404: 635-643.

14 Tjønneland A, Olsen A, Boll K, et al. Study design, exposure variables, and socioeconomic determinants of participation in Diet, Cancer and Health: a population-based prospective cohort study of 57,053 men and women in Denmark. Scand J Public Health 2007; 35: 432-441.
15 Carstensen B, Kristensen JK, Ottosen P, et al. The Danish National Diabetes Register: trends in incidence, prevalence and mortality. Diabetologia 2008; 51: 2187-2196.

16 Charlson ME, Pompei P, Ales KL, et al. A new method of classifying prognostic comorbidity in longitudinal studies: development and validation. J Chronic Dis 1987; 40: 373-383.

17 Chiolero A, Faeh D, Paccaud F, et al. Consequences of smoking for body weight, body fat distribution, and insulin resistance. Am J Clin Nutr 2008; 87: 801-809.

18 Tolstrup JS, Heitmann BL, Tjønneland AM, et al. The relation between drinking pattern and body mass index and waist and hip circumference. Int J Obes (Lond) 2005; 29: 490-497.

19 Wang Y, Rimm EB, Stampfer MJ, et al. Comparison of abdominal adiposity and overall obesity in predicting risk of type 2 diabetes among men. Am J Clin Nutr 2005; 81: 555-563.

20 Rothman KJ. BMI-related errors in the measurement of obesity. Int J Obes (Lond) 2008; 32: Suppl. 3, S56-S59.

21 Kornum JB, Thomsen RW, Riis A, et al. Diabetes, glycaemic control, and risk of hospitalization with pneumonia: a populationbased case-control study. Diabetes Care 2008; 31: 1541-1545.

22 Koziel H, Koziel MJ. Pulmonary complications of diabetes mellitus. Pneumonia. Infect Dis Clin North Am 1995; 9: 65-96.

23 Beuther DA, Sutherland ER. Overweight, obesity, and incident asthma: a meta-analysis of prospective epidemiologic studies. Am J Respir Crit Care Med 2007; 175: 661-666.

24 Talbot TR, Hartert TV, Mitchel E, et al. Asthma as a risk factor for invasive pneumococcal disease. N Engl J Med 2005; 352: 2082-2090.

25 Jackson ML, Neuzil KM, Thompson WW, et al. The burden of community-acquired pneumonia in seniors: results of a population-based study. Clin Infect Dis 2004; 39: 1642-1650. 